\title{
Ultrafast coherent control of angular momentum during a one-photon excitation
}

\author{
D. A. Malik, ${ }^{*}$ A. T. J. B. Eppink, W. L. Meerts, A. V. Kimel, A. Kirilyuk, Th. Rasing, and W. J. van der Zande \\ Radboud University Nijmegen, Institute for Molecules and Materials, P.O. Box 9010, NL-6500 GL Nijmegen, The Netherlands
}

(Received 23 December 2010; published 4 October 2011)

\begin{abstract}
The subpicosecond dynamics of angular momentum transfer in the excited rubidium $5 p$ state is studied in real time by observing photoelectron angular distributions with velocity map imaging. Retrieving the populations of the degenerate Zeeman levels and reconstructing the angular momentum, we show that in the case of resonant excitation the angular momentum does not follow the momentary helicity of the electric field of the pulse. This is in contrast with off-resonant excitation where the angular momentum and pulse helicity are fully correlated. Our study shows how to generate and shape ultrashort pulses of orbital and spin angular momentum in a controllable way.
\end{abstract}

DOI: 10.1103/PhysRevA.84.043404

PACS number(s): $32.80 . \mathrm{Qk}, 32.80 . \mathrm{Wr}$

\section{INTRODUCTION}

Laser excitation is one of the main approaches for manipulation of spins in solid-state quantum computing and semiconductor spintronics [1-5]. Optical pumping of spins is normally based on the absorption of circularly polarized photons and angular momentum transfer from photons to electrons. Due to the conservation of angular momentum and the spin-orbit interaction, such an excitation can result in an effective generation of a nonequilibrium spin polarization [6]. Over the last 20 years the spin dynamics at the nanosecond and picosecond time scale following optical excitation has been intensively studied and is quite well understood [7]. However, how the angular momentum evolves during the excitation is a question yet to be answered. Although optical manipulation of spins in atoms by optical pumping has been known for more than 50 years [8,9], even for an ideal system such as an atomic gas, the real-time dynamics of the angular momentum during action of a short laser pulse has remained unexplored for a long time. The advances in solving this problem have been made in gas phase alkalis with the recent developments in control and monitoring the interaction with light [10-13]. Photoelectron angular distributions (PADs) allow direct monitoring of the electron wave function of the photoelectrons and indirect monitoring of the intermediate states; also the angular momentum can be reconstructed [13]. In this paper, we combine the ultrafast pump-probe technique and polarization pulse shaping together with PAD measurements using velocity map imaging (VMI) in order to study and control the angular momentum dynamics on a 100-fs time scale using atomic $\mathrm{Rb}$ as a model system. We show that in a one-photon transition to the degenerate $5 p_{3 / 2}$ Zeeman levels the spectral properties of the excitation pulse play a crucial role. If resonant photons are presented in the pulses, the angular momentum in the excited state does not follow the helicity of the excitation pulse. Upon removal of resonant photons, the angular momentum follows the helicity of the excitation pulse.

\section{EXPERIMENT}

In the experiment, amplified 40-fs laser pulses were used with a repetition rate of $250 \mathrm{kHz}$, generated from a Coherent

\footnotetext{
*d.malik@science.ru.nl
}

Reg A 9050 amplifier. The wavelength of the radiation was centered around $795 \mathrm{~nm}$. The energy of the pulse was $\sim 4 \mu \mathrm{J}$. In Fig. 1(a) the pump-probe scheme is shown. A part of the laser beam (60\%) was used for the excitation and shaped using a pulse shaper with a so-called $4 f$ geometry, as described in Präkelt et al. [14]. Details of the shaper can be found in [15]. As control parameters, we rotated the polarization of part of the spectrum by $90^{\circ}$ (referred to as the polarization step) in combination with amplitude variations of part of the spectrum or an extra phase difference between the orthogonally polarized frequencies. The pulse shaping lengthens the excitation pulse to many picoseconds. The probe ionization pulses at $400 \mathrm{~nm}$ were obtained by second-harmonic generation in a BBO crystal by the unshaped fundamental pulses and were experimentally verified to have a duration of $100 \mathrm{fs}$. In a pump-probe experiment, the angular momentum in the excited state was determined as a function of time delay between the excitation and ionization pulses.

The schematic layout of the experiment is presented in Figs. 1 and 1(b). A diffuse flux of rubidium atoms was produced from an oven held at $60{ }^{\circ} \mathrm{C}$ and positioned $\sim 5 \mathrm{~cm}$ from the interaction region. During the experiment the pressure in the vacuum chamber was always below $10^{-6}$ mbar. The repeller and extractor plates act as a velocity map imaging lens for the photoelectrons. VMI creates a direct correspondence between the velocity vector of an electron and the position on an image [16]. The photoelectrons were detected by a double microchannel plate (MCP) detector combined with a phosphor screen and images were recorded with a CCD camera.

In order to control the angular momentum of $\mathrm{Rb}$ we employ the resonant $5 s_{1 / 2}-5 p_{3 / 2}$ transition at $\lambda_{i g}=780.2 \mathrm{~nm}\left(h \omega_{i g}=\right.$ $1.59 \mathrm{eV}$ ) [see Fig. 1(c)]. It is convenient to choose the propagation direction of the light as the quantization axis, implying that linearly polarized spectral components are superpositions of circularly polarized waves $[12,17]$ and only the Zeeman sublevels $m_{L}=1$ and $m_{L}=-1$ are coherently excited. In our computations, the $\mathrm{Rb} 5 p_{3 / 2} m$ states are described as $\left|L, m_{L}\right\rangle$ eigenstates, ignoring electron spin, or using the uncoupled representation $\left|L, m_{L}, S, m_{s}\right\rangle$. In our experiment, we control the magnitude of orbital angular momentum along the propagation direction of the light. The control variable is the product of the $5 p$ population and the expectation value of $L_{z}$ and is 

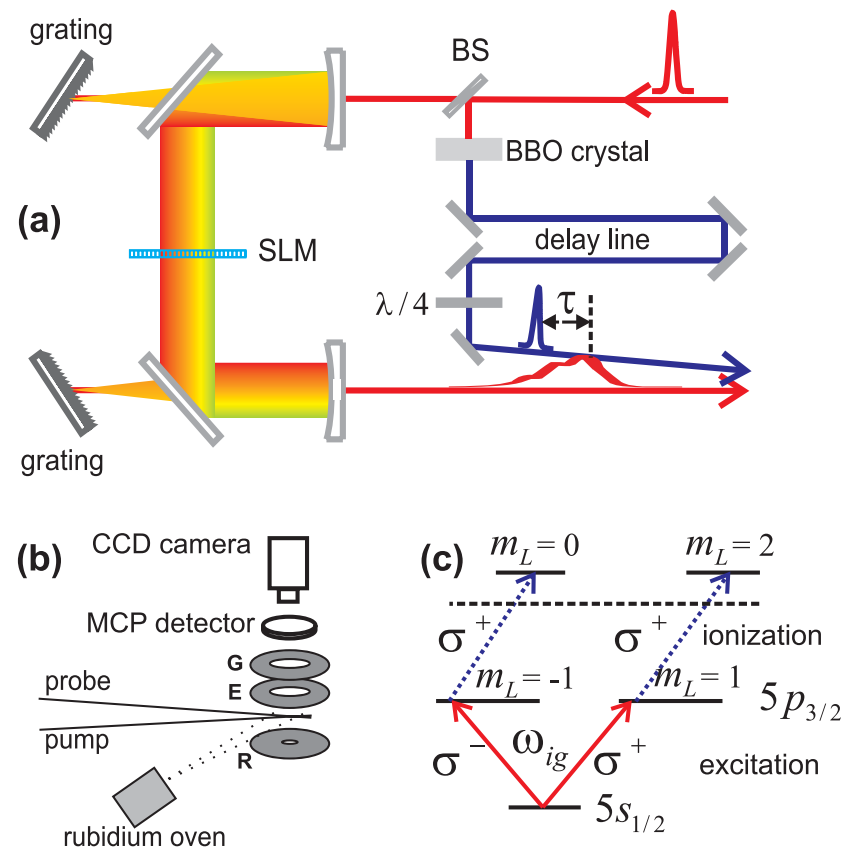

(d)

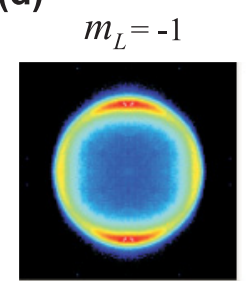

populated states:
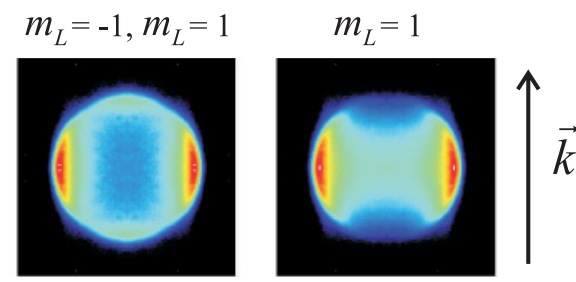

FIG. 1. (Color) (a) Pump-probe scheme. Part of the laser beam after the beam splitter (BS) is shaped in a pulse shaper with a computer-controlled spatial light modulator (SLM). The probe ionization pulses at $400 \mathrm{~nm}$ are obtained by second-harmonic generation in a BBO crystal by the unshaped fundamental pulses. The angular momentum in the excited state is determined as a function of time delay between the excitation and ionization pulses. (b) Schematic layout of the experiment. The angular momentum in the $5 p$ state is probed via ultrafast photoionization, using velocity map imaging. $\mathrm{R}$, $\mathrm{E}$, and $\mathrm{G}$ are the repeller, extractor, and ground plates respectively. (c) The excitation and the probe level scheme. (d) The experimental calibration PADs, obtained during the excitation with circularly and linearly polarized pulses, resulting in $\left\langle L_{z}\right\rangle=1$ (only $m_{L}=-1$ level populated), 0 (both $m_{L}=-1$ and $m_{L}=1$ levels are populated), and $-1\left(m_{L}=1\right.$ level is populated), respectively. The laser beam propagates in the vertical direction.

given by

$$
\begin{gathered}
\left\langle a_{1} Y_{1}^{1}(\theta, \varphi)+a_{-1} Y_{1}^{-1}(\theta, \varphi)\left|L_{z}\right| a_{1} Y_{1}^{1}(\theta, \varphi)\right. \\
\left.\quad+a_{-1} Y_{1}^{-1}(\theta, \varphi)\right\rangle \propto\left|a_{1}\right|^{2}-\left|a_{-1}\right|^{2}
\end{gathered}
$$

where $a_{m}$ is the probability amplitude of the corresponding $m$ state and $Y_{l}^{m}(\theta, \varphi)$ are the spherical harmonics. It can easily be shown that the expectation values of $\left\langle L_{x}\right\rangle$ and $\left\langle L_{y}\right\rangle$ are zero at all times.

By employing circularly polarized pulses in the ionization step [see Fig. 1(c)] we distinguish between the contributions from the $m_{L}=-1$ and the $m_{L}=1$ sublevels in the PAD images. In Fig. 1(d) experimental calibration images are shown, obtained by using circularly or linearly polarized pulses with the spectrum blocked above $784 \mathrm{~nm}$. The laser beam propagates in the vertical direction in the image plane. These images have been symmetrized in the plane and normalized to maximum intensity after background subtraction. The reference PADs do not depend on the time delay between pump and probe pulse in the calibration experiments. The first PAD represents $\mathrm{Rb}$ atoms with the angular momentum pointing antiparallel to the quantization axis, which implies $\left\langle L_{z}\right\rangle=-1$. The second image shows the PAD during the excitation by linearly polarized pulse, where the angular momentum is perpendicular to the quantization axis, with $\left\langle L_{z}\right\rangle=\left\langle L_{x}\right\rangle=\left\langle L_{y}\right\rangle=0$. The difference in intensities around the vertical nodes $\left(m_{L}=-1\right)$ and horizontal nodes $\left(m_{L}=1\right)$ is due to the difference in strength in the ionization step. The third image shows the case of angular momentum parallel to the quantization axis, $\left\langle L_{z}\right\rangle=1$. The different symmetries of the photoelectron angular distributions reflect directly the angular momentum in the $5 p$ excited state. From the images, we retrieve the populations of the Zeeman levels and deduce $\left\langle L_{z}\right\rangle$.

In our retrieval procedure the angular part of the electronic wave function in the continuum is described as a coherent superposition of angular momentum states via spherical harmonics $Y_{m}^{l}(\theta, \varphi)$ :

$$
\psi(\theta, \varphi)=\sum_{m, l^{\prime}} \mu_{m, m+1}^{l^{\prime}} a_{m} Y_{m+1}^{l^{\prime}}(\theta, \varphi) e^{-i \xi_{l \prime}},
$$

where $m=m_{L}=-1,1$ are the possible Zeeman levels in the intermediate $5 p_{3 / 2}$ level. The coefficients $a_{m}$ are the transition probability amplitudes from the ground $5 \mathrm{~s}$ state to the excited $m_{L}$ states in the $5 p_{3 / 2}$ state. In the continuum, $l^{\prime}$ $=0,2$ are the final possible orbital angular momentum states, $\mu_{m, m+1}^{l^{\prime}}$ is the $m$-dependent transition dipole moment matrix element between excited state and final state with orbital momentum $l^{\prime}$, and $\xi_{l^{\prime}}$ is the asymptotic phase of the continuum wave function, as defined in [18]. The photoelectron angular distribution, measured in the experiment is the integral of $\psi(\theta, \varphi) \psi^{*}(\theta, \varphi)$ in the direction perpendicular to the image plane.

To extract the populations $a_{m} a_{m}^{*}$ of the $m_{L}=1$ and $m_{L}=-1$ sublevels we fit the experimental images with the previously described model [see Eq. (2)]. Because of the complexity of the transition probability amplitudes $a_{m}$, this requires a fit of four parameters including the real and imaginary parts of $a_{m}$. However, the photoelectron angular distribution $\psi(\theta, \varphi) \psi^{*}(\theta, \varphi)$ depends only on the relative phase between $a_{+1}$ and $a_{-1}$. Hence, we searched for three parameters in the fitting procedure: the absolute values of $a_{m}$ and the relative phase between $a_{+1}$ and $a_{-1}$. In the fitting procedure the intensities in the image were stored line by line in a one-dimensional vector array and the inner product between the experimental and calculated vectors was maximized using an evolutionary algorithm (Evolution Strategy) [19]. In our fitting procedure the Covariance Matrix Adaptation Evolution Strategy (CMA-ES) algorithm developed by Hansen and Ostermeier was used [20]. In the model [see Eq. (2)] parameters characterizing outgoing channels with the $s$ and 


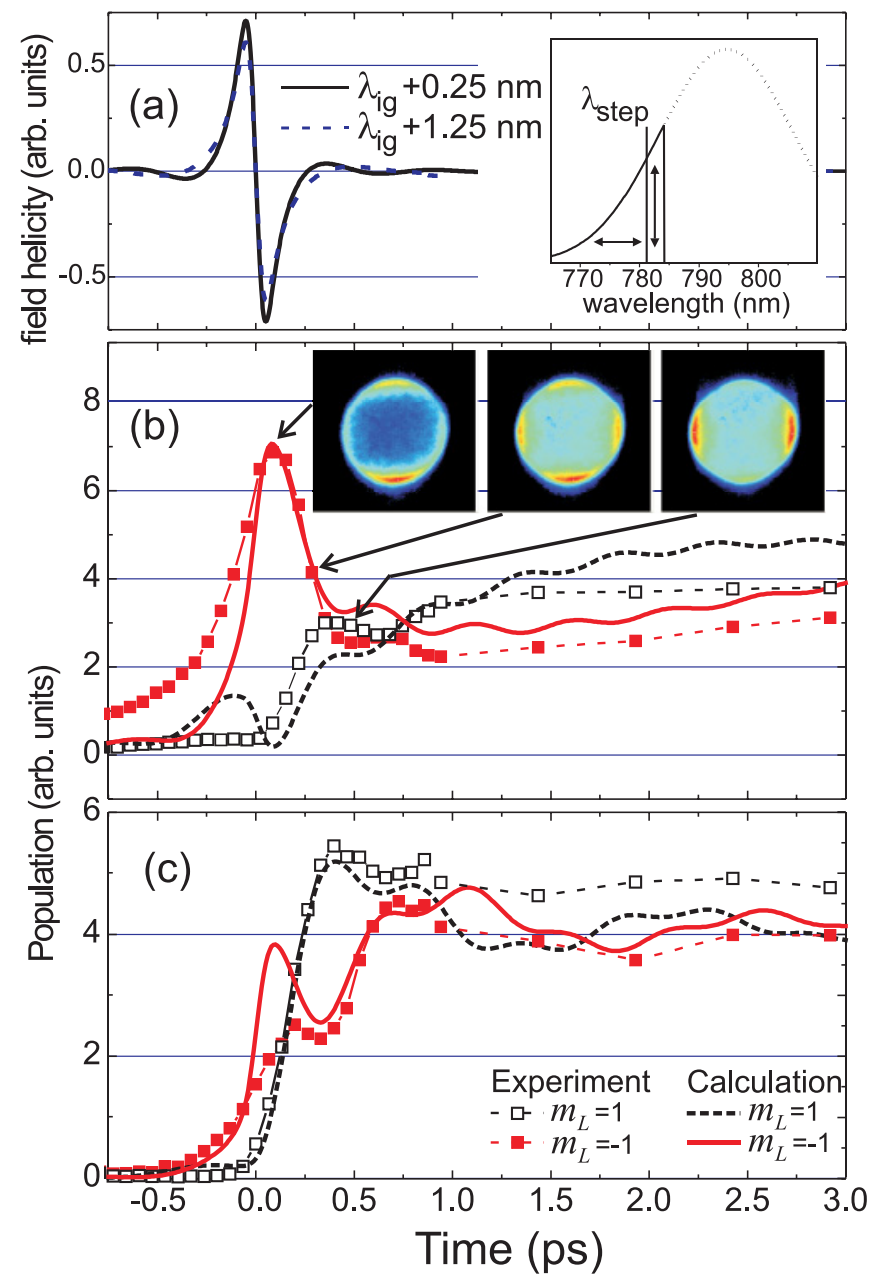

FIG. 2. (Color) (a) Temporal evolution of pulse field helicity $\vec{h}=i\left[\vec{E} \times \vec{E}^{*}\right]$ for the cases when the polarization step is applied at $\lambda_{i g}+0.25 \mathrm{~nm}$ (black solid line) and at $\lambda_{i g}+1.25 \mathrm{~nm}$ (blue dashed line). The inset shows the excitation spectrum. Part of the spectrum above $784 \mathrm{~nm}$ is blocked. (b) Experimental and calculated populations of the $m=1, m=-1$ sublevels as a function of time. Polarization step $\lambda_{\text {step }}$ is applied at $\lambda_{i g}+0.25 \mathrm{~nm}$. (c) The same as the data shown in (b) but for the case when the polarization step is applied at $\lambda_{i g}+1.25 \mathrm{~nm}$.

$d$ character of the wave function were taken from [18]. The ratio of the strength between the $s$ and $d$ channels was set to the experimental value of 0.38 . The value of the asymptotic phase difference $\xi_{l^{\prime}}$ between the $s$ and $d$ channels was taken as $1.7 \mathrm{rads}$, close to the given value of 1.85 rads. For the optimum fit of the calibration images, obtained from the excitation with pure linearly and circularly polarized pulses a residual ellipticity of the ionization pulses and correction for the $\xi_{l^{\prime}}$ had to be implemented.

We have also analyzed the PADs by taking electron spin into consideration. In those efforts, the coupled representation $\left|J, M_{J} ; L\right\rangle$ was used for analyzing the excitation step. The ionization step was always described in the uncoupled basis $\left|L, M_{L}\right\rangle$. We found that inclusion of electron spin and the spin-orbit coupling had little effect on the PADs. In fact, the calculated magnetization of our $\mathrm{Rb}$ samples follows accurately the angular momentum in the excited state.

\section{RESULTS AND DISCUSSION}

The retrieved populations of the $m_{L}=-1$ and $m_{L}=+1$ states are directly compared to numerical calculations of the populations from the time-dependent Schrödinger equation in the perturbative limit. In Fig. 2 the results are shown when a polarization step was positioned at $\lambda_{\text {step }}=\lambda_{i g}+0.25 \mathrm{~nm}$ (corresponding to $\hbar \omega_{i g}=0.51 \mathrm{meV}$ ). Since we want to control the angular momentum dynamics within the degenerate Zeeman levels only, we want to avoid spin-orbit precession motion related to the coherent excitation of both $5 p_{3 / 2}$ and $5 p_{1 / 2}$ states [11]. Therefore, we reduced the transient population in the $5 p_{1 / 2}$ level relatively to the population in the $5 p_{3 / 2}$ level by approximately two orders of magnitude by blocking the spectrum above $784 \mathrm{~nm}$. The temporal profile of the shaped pulse has a complicated form; in Fig. 2(a) the time evolution of the helicity of the field of the pulse $\vec{h}=i\left[\vec{E} \times \vec{E}^{*}\right]$ is shown. The intense part of the pulse has a duration of $250 \mathrm{fs}$ (full width at half maximum), during which the helicity changes within 100 fs. A sudden change in polarization creates long pre- and post-transients in the pulse, characterized by a periodic reversal of the helicity. The populations of the $m_{L}=-1$ and $m_{L}=+1$ states of $\mathrm{Rb}$ atoms are shown in Fig. 2(b). Initially, $\left\langle L_{z}\right\rangle=0$ at the rising edge of the pulse and then the angular momentum grows in the direction antiparallel to the propagation, as mostly the $m_{L}=-1$ state gets populated within 200 fs. The difference in population dynamics between the $m$ levels during the intense part of the pulse reflects the response of the system on a real-time helicity. While the intensity of the pulse decreases strongly after $1 \mathrm{ps}$, still significant population changes are observed and $\left\langle L_{z}\right\rangle$ changes sign without reaching the maximum value found at $100 \mathrm{fs}$. At a longer time scale the population difference between the two Zeeman levels asymptotically disappears and the excited atoms are no longer polarized. The minor oscillations with a period of $520 \mathrm{fs}$ follow the periodic helicity reversal of the weak post-transient.

The next experiment corroborates the absence of a direct relation between the helicity and the observed direction of the angular momentum vector. We have varied the position of the polarization step with respect to the resonance of the one-photon transition from $\lambda_{i g}+0.25 \mathrm{~nm}$ to $\lambda_{i g}+$ $1.25 \mathrm{~nm}$. In the intense part of the pulse from -250 to 250 fs helicity remains almost unchanged, while the angular momentum vector is already inverted at 200 fs [compare Figs. 2(b) and 2(c)]. The period of the angular momentum vector reversal as well as the decay time of $\left\langle L_{z}\right\rangle$ during the transients decrease when the polarization step is applied further from resonance. This systematic dependence of the angular momentum on polarization step position is shown in Fig. 3. Our interpretation of the angular momentum reversal dynamics is as follows. In the perturbative limit long after the pulse, the populations in both magnetic sublevels are determined only by the resonant frequency component in the spectrum. At short excitation times a broad spectral range of frequencies contributes. Effectively, the excitation time determines the spectral width around the resonance, that contributes to the excitation, hence a faster decay of the angular momentum is observed at larger detunings of the polarization step from the resonance. We note that the slight offset between the 


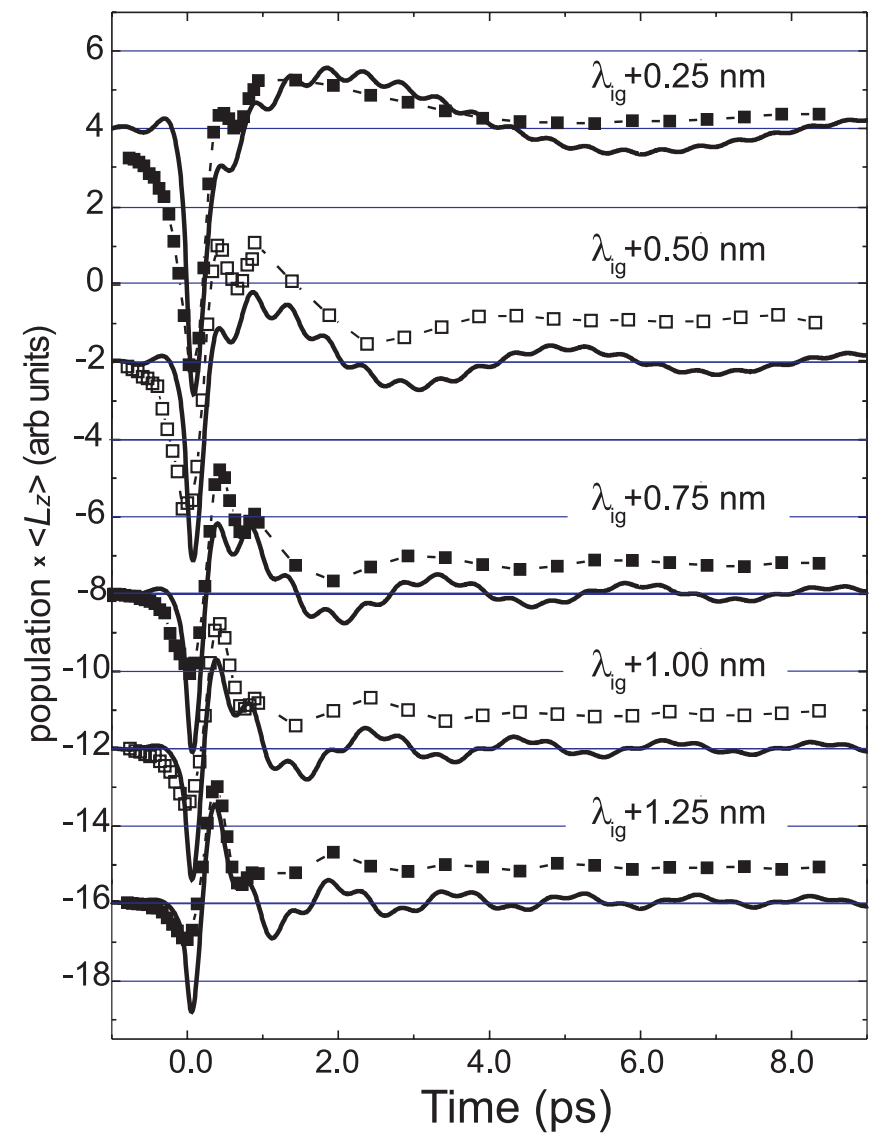

FIG. 3. (Color online) Experimental and calculated product of population and expectation value $\left(\left|a_{1}\right|^{2}+\left|a_{-1}\right|^{2}\right)\left\langle L_{z}\right\rangle$ as a function of the polarization step position for conditions as in Fig. 2.

calculations and the experimental data in Fig. 3 is due to a small systematic underestimation of one of the Zeeman levels in the analysis. The reason for this might be that the evolutionary algorithm introduces a bias due to imperfect background corrections and due to the symmetrization of images. Of course, our excitation pulse is affected by the nonideality of the pulse shaper [21].

The dynamics of the angular momentum is affected by interference between resonant and off-resonant excitation to a large extent. As it was shown in [22] using chirped pulses, the interference results in periodic oscillation dynamics of the population in the excited state. The sharp changes in polarization in the spectrum result in a similar oscillatory behavior of the populations in the $m_{L}=+1$ and $m_{L}=-1$ sublevels and creation of angular momentum. We note that the period of helicity changes in the transient is determined by the inverse spectral width between the polarization step and the spectral block (784 nm). This explains the absence of any correlation between helicity and angular momentum reversal during the transients.

Removal of the resonant wavelength prevents interference to occur, allowing off-resonant excitation only. In the next experiment the excitation spectrum was limited to the region from $780.7 \mathrm{~nm}$ to $784 \mathrm{~nm}$ and the polarization step was positioned at $782.5 \mathrm{~nm}$. The experimental and calculated values of $\left\langle L_{z}\right\rangle$ are shown in Fig. 4(b). By removing the resonant frequencies, the excited state population is only

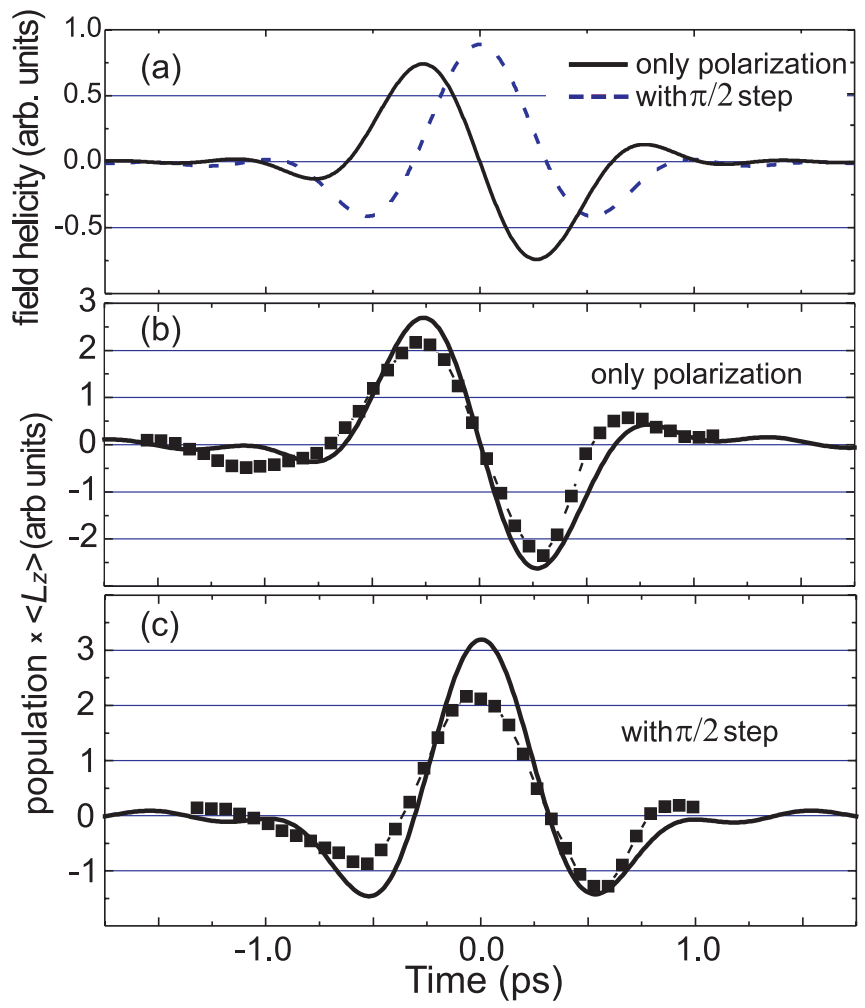

FIG. 4. (Color online) Ultrafast evolution of angular momentum during off-resonant excitation. The excitation spectrum is continuous from $780.7 \mathrm{~nm}$ to $784 \mathrm{~nm}$. The polarization step is applied at $782.5 \mathrm{~nm}$. (a) Temporal evolution of the pulse field helicity $\vec{h}=i\left[\vec{E} \times \vec{E}^{*}\right]$ for the pulses having only the polarization step (black solid line) and the polarization step together with $\pi / 2$ phase step at $782.5 \mathrm{~nm}$ (blue dashed line). (b) Experimental and calculated product of population and expectation value $\left(\left|a_{1}\right|^{2}+\left|a_{-1}\right|^{2}\right)\left\langle L_{z}\right\rangle$ for the pulse containing polarization step only. (c) The same as the data in (b) but for the case when the spectrum has both polarization step and $\pi / 2$ phase step at $782.5 \mathrm{~nm}$.

created transiently during the laser pulse and the angular momentum vanishes after 1 ps due to the depopulation of the excited state. In strong fields, where saturation is reached, an absence of resonant photons may still give an asymptotic population as has been shown before [23]. We observe that the angular momentum now fully correlates with the helicity of the pulse [see Fig. 4(a)]. The angular momentum has two major and two minor maxima of opposite sign. When an additional $\pi / 2$ phase step is applied in the spectrum, the resulting dynamics still correlates with the momentary helicity of the pulse. In Fig. 4(c) it is shown that the angular momentum has two maxima of one sign and one larger maximum of opposite sign. The fact that the angular momentum dynamics is much more easily predictable in the case of off-resonant ultrafast excitation is ascribed to the fact that off-resonant photons cannot result in a long-lived population of the excited state, i.e., the excited population is constantly refreshed following the real-time pulse.

\section{CONCLUSION}

In conclusion, we have demonstrated ultrafast coherent control of the orbital angular momentum in one-photon 
transitions by manipulating the excitation to the degenerate Zeeman levels. We have shown that also in a one-photon transition at ultrashort times coherent excitation by frequencies with different polarization states results in a complex polarization response of a system. This response does not follow the polarization of the excitation pulse in a straightforward manner. Our results indicate that it is possible to generate ultrashort pulses of orbital angular momentum and, in a similar way, probably of magnetization in samples, as well as periodic angular momentum reversal. Our results give insight into the ultrafast control of angular momentum in optical excitation and may provide an alternative to the ultrafast control of orbital and spin degrees of freedom.

\section{ACKNOWLEDGMENTS}

We would like to acknowledge the technical support of A. J. A. van Roij, T. Toonen, A. van Etteger, and the financial support of FOM, NWO, EU-Marie Curie ITN Program ICONIC, European Research Council (Grant No. FP7/2007-2013) N 257280. We also thank D. H. Parker for providing experimental equipment.
[1] D. Press, T. D. Ladd, B. Zhang, and Y. Yamamoto, Nature 456, 218 (2008).

[2] A. Greilich, S. E. Economou, S. Spatzek, D. R. Yakovlev, D. Reuter, A. D. Wieck, T. L. Reinecke, and M. Bayer, Nat. Phys. 5, 262 (2009).

[3] J. Berezovsky, M. H. Mikkelsen, N. G. Stoltz, L. A. Coldren, and D. D. Awschalom, Science 320, 349 (2008).

[4] C. Phelps, T. Sweeney, R. T. Cox, and H. Wang, Phys. Rev. Lett. 102, 237402 (2009).

[5] C. Le Gall, L. Besombes, H. Boukari, R. Kolodka, J. Cibert, and H. Mariette, Phys. Rev. Lett. 102, 127402 (2009).

[6] Yu. G. Kusrayev, Semicond. Sci. Technol. 23, 114013 (2008).

[7] Spin Physics in Semiconductors, edited by M. I. Dyakonov (Springer-Verlag, Berlin, 2008).

[8] A. Kastler, J. Opt. Soc. Am. 47, 460 (1957).

[9] W. Happer, Rev. Mod. Phys. 44, 169 (1972).

[10] D. Meshulach and Y. Silberberg, Phys. Rev. A 60, 1287 (1999).

[11] B. Chatel, D. Bigourd, S. Weber, and B. Girard, J. Phys. B: At. Mol. Opt. Phys. 41, 074023 (2008).

[12] M. Wollenhaupt, M. Krug, J. Köhler, T. Bayer, C. SarpeTudoran, and T. Baumert, Appl. Phys. B 95, 245 (2009).
[13] M. Wollenhaupt, M. Krug, J. Köhler, T. Bayer, C. SarpeTudoran, and T. Baumert, Appl. Phys. B 95, 647 (2009).

[14] A. Präkelt et. al., Rev. Sci. Instrum. 74, 4950 (2003).

[15] A. S. Meijer, A. V. Kimel, Th. Rasing, and W. J. van der Zande, Phys. Rev. A 78, 053403 (2008).

[16] A. T. J. B. Eppink and D. H. Parker, Rev. Sci. Instrum. 68, 3477 (1997).

[17] D. A. Malik, A. V. Kimel, A. Kirilyuk, Th. Rasing, and W. J. van der Zande, Phys. Rev. Lett. 104, 133001 (2010).

[18] Z.-M. Wang and D. S. Elliott, Phys. Rev. A 62, 053404 (2000).

[19] W. L. Meerts and M. Schmitt, Int. Rev. Phys. Chem. 25, 353 (2006).

[20] N. Hansen and A. Ostermeier, Evolutionary Computation 9(2), 159 (2001).

[21] B. J. Sussman, R. Lausten, and A. Stolow, Phys. Rev. A 77, 043416 (2008).

[22] J. Degert, W. Wohlleben, B. Chatel, M. Motzkus, and B. Girard, Phys. Rev. Lett. 89, 203003 (2002).

[23] A. Gürtler and W. J. van der Zande, Phy. Rev. Lett. 93, 153002 (2004). 